\title{
Right Insular Atrophy in Neurocardiogenic Syncope: A Volumetric MRI Study
}

\author{
J.B. Kim, S.-i. Suh, W.-K. Seo, S.-B. Koh, and J.H. Kim
}

\begin{abstract}
BACKGROUND AND PURPOSE: Alterations in the central autonomic network are hypothesized to play a role in the pathophysiologic mechanism underlying neurocardiogenic syncope; however, few data are available regarding the structural changes of the brain in this condition. We used voxel-based morphometry and regional volumetry to identify possible neuroanatomic correlates.
\end{abstract}

MATERIALS AND METHODS: We prospectively studied 32 patients with neurocardiogenic syncope with a positive response to the head-up tilt test and 32 controls who had no history of syncope. We used voxel-based morphometry to compare GM volumes between patients and controls. In addition, regional volumes of structures known to be included in the central autonomic network were measured and compared between the groups. Correlation analyses were also performed between clinical variables and anatomic data.

RESULTS: Voxel-based morphometry showed a significant GM volume reduction in the right insular cortex in patients with neurocardiogenic syncope compared with controls (corrected $P=.033$ ). Regional volumetry showed a significant reduction of right insular volumes in patients compared with controls $(P=.002$, MANCOVA). Smaller right insular volumes in patients with neurocardiogenic syncope were related to larger drops in systolic $(P=.020)$ and diastolic $(P=.003)$ blood pressures during the head-up tilt test.

CONCLUSIONS: We observed a novel finding of right insular atrophy in patients with neurocardiogenic syncope with a positive response to the head-up tilt test, implicating the role of right insular dysfunction in the pathophysiologic mechanism underlying neurocardiogenic syncope. Our findings further support the hypothesis that right insular dysfunction may cause a decrease in sympathetic activity and a reciprocal increase in parasympathetic activity, leading to syncope.

ABBREVIATIONS: $\mathrm{BP}=$ blood pressure; $\mathrm{HR}=$ heart rate; $\mathrm{HUT}=$ head-up tilt test; $\mathrm{NCS}=$ neurocardiogenic syncope; VBM = voxel-based morphometry

$\mathbf{N}$ eurocardiogenic syncope (NCS) (simple fainting or vasovagal syncope) is the most frequent cause of syncope. ${ }^{1,2}$ It refers to a condition in which autonomic cardiovascular reflexes become inappropriate in response to triggers, leading to transient cerebral hypoperfusion and loss of consciousness with spontaneous recovery. ${ }^{3-5}$ Although the pathophysiologic mechanism of NCS remains incompletely understood, paradoxic compensatory reflex has been suggested as the most probable mechanism. ${ }^{3,6}$

Received March 3, 2013; accepted after revision March 29

From the Departments of Neurology (J.B.K, W.-K.S., S.-B.K., J.H.K.) and Radiology (S.-i.S.) Korea University Guro Hospital, Korea University College of Medicine, Seoul, Korea.

This work was supported in part by the National Research Foundation of Korea Grant funded by the Korean Government (Grant No. 20100004827, 20110005418) and a Korea University Grant (to J.H. Kim). We certify that there is no conflict of interest.

Please address correspondence to Ji Hyun Kim, MD, PhD, Department of Neurology, Korea University Guro Hospital, Korea University College of Medicine, 152-

703, Guro-dong Ro 148, Guro-gu, Seoul, Republic of Korea; email: jhkim.merrf@gmail.com

- Indicates open access to non-subscribers at www.ajnr.org

http://dx.doi.org/10.3174/ajnr.A3611
Reduction of cardiac ventricular volume activates baroreceptor-mediated sympathetic outflow, which causes vigorous contraction of an empty cardiac ventricle. In turn, activation of mechanoreceptors in the ventricular wall might trigger an inhibitory response through increasing vagal tone, resulting in paradoxic bradycardia and hypotension., 3 This widely accepted "ventricular theory " basically includes increased parasympathetic activity caused by alteration of autonomic controls in the CNS. ${ }^{7}$ Therefore, the understanding of CNS structures involved in the autonomic control system might be crucial for elucidating fundamental mechanisms of NCS.

To our knowledge, there is 1 voxel-based morphometry (VBM) study that investigated changes in brain structures in patients with NCS. ${ }^{8}$ VBM is an objective and fully validated method, which can detect regionally specific differences in GM structures on a voxelwise comparison between groups. ${ }^{9}$ In the present study, we applied VBM and regional volumetry to identify the structural GM changes associated with NCS. In addition, we investigated possible relationships between the structural changes and clinical variables such as disease duration, frequency of syncope attacks, and blood pressure (BP) and heart rate (HR) changes during head-up tilt test (HUT). 


\section{MATERIALS AND METHODS Subjects}

Consecutive patients with NCS were prospectively recruited from the outpatient clinic at the Neurology Department, Korea University Guro Hospital, from January 2010 to April 2012. Inclusion criteria were as follows: 1) unequivocal symptoms of syncope (defined as transient loss of consciousness and postural tone with spontaneous recovery) and presyncope (defined as a state of lightheadedness, muscular weakness, and feeling faint without complete loss of consciousness); 2) orthostatic stress as the only trigger of syncope and a positive response to HUT (as described below); 3) no history of syncope relevant to situational syncope or carotid sinus hypersensitivity; 4) absence of any abnormal findings on electroencephalography; 5) no history of epileptic seizure; 6) no evidence of developmental and neurologic abnormalities; 7) neither abnormal nor unusual findings on conventional MR imaging; 8) no evidence of cardiogenic syncope based on electrocardiography and echocardiography; 9) no history of diabetes, parkinsonism, and peripheral neuropathy; and 10) no history of significant head injury, alcohol or psychotropic drug abuse, or neurologic and psychiatric disorders. Demographic and clinical data such as age of onset, disease duration, and the number of syncope and presyncope attacks were obtained through interviews with the patients and their family members and reviews of medical records.

For group comparison, 32 right-handed healthy volunteers matched for age and sex were recruited to serve as control subjects. All control subjects underwent neurologic examination and a detailed interview to ensure that they did not have the following: 1) a history of syncope and presyncope; 2) a history of headache disorders, significant head injury, neurologic, psychiatric, or systemic disorders; and 3) a history of alcohol or psychotropic drug abuse. The local ethics committee approved the study protocol, and all participants gave written informed consent before study inclusion.

\section{Head-Up Tilt Test}

None of the patients were on any medications at the time of the HUT examination. Orthostatic BP recording was performed with a sphygmomanometer cuff over the brachial artery and a monitor console displaying systolic and diastolic BP. Simultaneous HR recording was performed as well. After a 20-minute rest in the supine position and measurement of baseline BP and HR, each subject was positioned at an angle of $70^{\circ}$ from the supine position for as long as 45 minutes or until syncope occurred on a standard electrically driven tilt table with a footboard. Serial measurement of BP and HR was performed every 2 minutes during HUT. Pharmacologic provocation was not performed. ${ }^{10}$ The response to HUT was defined as positive when the development of symptoms recognized by the patients was similar to those of spontaneous syncope in association with hypotension, bradycardia, or both. ${ }^{4,11,12}$ As soon as syncope occurred, the patient was rapidly returned to the supine position. Changes in BP and HR were measured from the baseline to the onset of the syncope attack.

\section{MR Imaging Acquisition}

MR images were acquired on a Trio 3T scanner (Siemens, Erlangen, Germany) with a 12-channel phased array head coil. For volumetric analysis, a high-resolution 3D MPRAGE sequence was acquired with the following parameters: $\mathrm{TR}=1780 \mathrm{~ms}$, $\mathrm{TE}=2.34 \mathrm{~ms}$, matrix $=$ $256 \times 256, \mathrm{FOV}=256 \times 256 \mathrm{~mm}$, voxel dimension $=1 \mathrm{~mm}^{3}$. For identification of structural abnormalities, the following conventional MR images were acquired: axial T2-weighted and FLAIR images (4-mm thickness), oblique coronal T2-weighted and FLAIR images perpendicular to the long axis of the hippocampus (3-mm thickness), and gadolinium-enhanced axial T1-weighted images (5-mm thickness). The MR images of all participants were reviewed by a board-certified neuroradiologist (S.-i.S.) for any structural abnormalities, and findings were reported as normal.

\section{Voxel-Based Morphometry}

Data were processed and analyzed by using SPM8 (Wellcome Department of Imaging Neuroscience, London, UK), where we applied VBM implemented in the VBM8 toolbox (http://dbm. neuro.uni-jena.de/vbm.html) with default parameters. Images were bias-corrected, tissue-classified, and registered by using linear (12-parameter affine) and nonlinear transformations, within a unified segmentation model. ${ }^{13}$ Subsequently, analyses were performed on the volumes of the GM segments, which were multiplied by nonlinear components derived from the normalization matrix to compensate for possible volume changes during the nonlinear spatial normalization procedure. These modulated GM images were then smoothed with an 8-mm full width at half maximum isotropic Gaussian kernel. The analyses of modulated GM images can detect regional differences in GM volume.

Regionally specific differences in GM volume between patients with NCS and control subjects were assessed by using ANCOVA with total intracranial volume, age, and sex as nuisance variables. An absolute GM threshold of 0.2 (of a maximum value of 1) was used to avoid possible edge effects around the border between GM and WM. Two contrasts were defined to examine both increases and reductions in GM volume between the groups. Statistical significance was set at a height threshold of $P<.001$ and an extent threshold of cluster-level $P<.05$, corrected for multiple comparisons by using family-wise error.

\section{Regional Volumetry}

Cortical reconstruction and volumetric segmentation were performed by using FreeSurfer software (Version 5.1.0, http://surfer. nmr.mgh.harvard.edu). The technical details of the procedures are described elsewhere. ${ }^{14-17}$ Briefly, image preprocessing procedures included removal of nonbrain tissue, ${ }^{17}$ automated Talairach transformation, segmentation of the subcortical WM and deep GM volumetric structures, ${ }^{15}$ intensity normalization, tessellation of the GM/WM boundary, automated topology correction, ${ }^{18}$ and surface deformation to detect GM/WM and GM/CSF boundaries. Subsequent to cortical reconstruction, the cortex was subdivided into units on the basis of gyral and sulcal structures. ${ }^{19}$ This parcellation method was shown to be valid and reliable, with high intraclass correlation coefficients between the manual and automated procedures for volume measurement of 34 cortical regions of interest. ${ }^{19} \mathrm{~A}$ volume at each vertex was computed as the average area of the triangles around the vertex multiplied by the thickness at the vertex. The volume of a parcellated cortex was then computed as the sum of the volumes of the vertices in each parcellation. 
Demographic data, clinical characteristics, and regional volumetry of patients with NCS and controls ${ }^{\mathrm{a}}$

\begin{tabular}{lccc}
\hline & $\begin{array}{c}\text { Patients with NCS } \\
(\boldsymbol{n}=\mathbf{3 2})\end{array}$ & $\begin{array}{c}\text { Controls } \\
(\boldsymbol{n}=\mathbf{3 2})\end{array}$ & $\boldsymbol{P}$ Value \\
\hline Clinical features & & & \\
Age (yr) & $24.1 \pm 6.9$ & $24.8 \pm 5.2$ & .655 \\
Male sex (No.) (\%) & $11(34.4)$ & $12(37.5)$ & .794 \\
Age of onset (yr) & $20.8 \pm 7.1$ & & \\
Disease duration (yr) & $4.3 \pm 4.7$ & & \\
No. of total syncope and presyncope attacks & $38.4 \pm 74.4$ & & \\
Head-up tilt test findings & & & \\
Baseline systolic BP (mm Hg) & $109.5 \pm 12.2$ & & \\
Baseline diastolic BP (mm Hg) & $62.0 \pm 9.6$ & & \\
Baseline mean HR (beats/min) & $67.7 \pm 10.0$ & & \\
Change in systolic BP (mm Hg) & $-40.5 \pm 15.3$ & & \\
Change in diastolic BP (mm Hg) & $-18.3 \pm 10.1$ & & \\
Change in HR (beats/min) & $-33.5 \pm 15.8$ & & \\
Volume of structure (mm $\left.{ }^{3}\right)$ & & & \\
R rostral anterior cingulate & $2288 \pm 416.3$ & $2461 \pm 425.2$ & .137 \\
R caudal anterior cingulate & $2386 \pm 555.6$ & $2488 \pm 639.3$ & .498 \\
R insula & $7323 \pm 689.1$ & $8011 \pm 932.5$ & .002 \\
R amygdala & $1755 \pm 232.2$ & $1829 \pm 184.9$ & .339 \\
L rostral anterior cingulate & $2791 \pm 484.6$ & $3141 \pm 589.7$ & .014 \\
L caudal anterior cingulate & $1819 \pm 494.8$ & $2142 \pm 711.5$ & .063 \\
L insula & $7234 \pm 649.5$ & $7657 \pm 701.0$ & .018 \\
L amygdala & $1770 \pm 216.2$ & $1816 \pm 229.1$ & .963 \\
\hline
\end{tabular}

Note:- $R$ indicates right; L, left.

a The values are presented as means. An independent $t$ test and $\chi^{2}$ test were used to compare between-group differences in age and sex, respectively. MANCOVA adjusting for age,sex, and estimated total intracranial volume was performed to compare regional volumes of each structure between the groups.

The automated procedures for volumetric measurements of the subcortical GM structures were described in detail previously. ${ }^{15}$ Briefly, this procedure automatically provided segments and labels for up to 40 unique structures and assigned a neuroanatomic label to each voxel in an MR imaging volume on the basis of probabilistic information estimated automatically from a manually labeled training set. A Bayesian segmentation procedure was then performed, and the maximum a posteriori estimate of the labeling was computed. The segmentation uses 3 pieces of information to disambiguate labels: 1) the prior probability of a given tissue class occurring at a specific atlas location, 2) the likelihood of the image intensity given that tissue class, and 3) the probability of the local spatial configuration of labels given the tissue class. This technique has previously been shown to be comparable in accuracy with manual labeling. ${ }^{15}$ All segmentations were visually inspected for accuracy before inclusion in the analysis.

Among the automatically segmented regions, we focused on 3 cortical regions (rostral anterior cingulate, caudal anterior cingulate, and insula) and 1 subcortical region (amygdala) in each hemisphere, which are known to be parts of the central autonomic network. ${ }^{20-22}$ Volumes of the 8 regions were automatically measured and compared between patients and controls by using MANCOVA, adjusting for age, sex, and estimated total intracranial volume. Post hoc univariate tests with a Bonferroni correction were performed to follow the significant main effects yielded by the MANCOVA. Statistical significance was set at $P<.00625$ $(.05 / 8)$. Correlation analyses were performed between the normalized volumes of 8 structures (each regional GM volume corrected for estimated total intracranial volume) and clinical parameters such as disease duration, total frequency of syncope and presyncope, and BP and HR changes during HUT (Spearman correlation analysis, $P<.05)$. Statistical analyses were performed by using the Statistical Package for Social Sciences (Version 19.0; IBM, Armonk, New York).

\section{RESULTS \\ Clinical Characteristics}

Thirty-two right-handed patients and 32 controls were enrolled in this study. Clinical characteristics and the results of HUT are summarized in the Table. There were no differences in age and sex between patients and controls. The mean age of onset was $20.8 \pm 7.1$ years (range, 11-39 years), the mean duration of NCS was $4.3 \pm 4.7$ years (range, $1-22$ years), and the mean number of total syncope and presyncope attacks (mean number of attacks per year $\times$ disease duration in years) was $38.4 \pm 74.4$ (range, 2-300).

All patients showed positive responses to HUT. Mean baseline systolic BP was $109.5 \pm 12.2 \mathrm{~mm} \mathrm{Hg}$ (range, 87-148 mm $\mathrm{Hg}$ ), mean baseline diastolic BP was $62.0 \pm 9.6 \mathrm{~mm} \mathrm{Hg}$ (range, $47-81 \mathrm{~mm} \mathrm{Hg}$ ), and mean baseline HR was $67.7 \pm 10.0$ beats/min (range, $52-86$ beats/min). During HUT, mean change of systolic BP was $-40.5 \pm 15.3 \mathrm{~mm} \mathrm{Hg}$ (range, -16 to $-81 \mathrm{~mm} \mathrm{Hg}$ ), mean change of diastolic BP was $-18.3 \pm 10.1 \mathrm{~mm}$ $\mathrm{Hg}$ (range, 0 to $-36 \mathrm{~mm} \mathrm{Hg}$ ), and mean change of $\mathrm{HR}$ was $-33.5 \pm$ 15.8 beats/min (range, -5 to -65 beats/min). According to the Vasovagal Syncope International Study (VASIS) classification, ${ }^{23} 31$ of 32 patients had a type 1 mixed response and 1 patient had a type 3 vasodepressor response.

\section{Voxel-Based Morphometry and Regional Volumetry}

VBM showed a significant GM volume reduction in the right insular cortex in patients with NCS compared with control subjects (family-wise-error-corrected $P=.033$; cluster size $=1178$ voxels [ $3976 \mathrm{~mm}^{3}$ ]; peak $z$ score $=4.06$; Montreal Neurological Institute coordinates of local maxima $=42,3,-6$; resolution element count $=1.5)($ Fig 1$)$. No region of a significant GM volume increase was found in patients compared with controls at the same threshold (family-wise-error-corrected $P<.05$ ).

Regional volumes of 8 GM structures are presented in the Table. Volumes of the right insular cortex were significantly reduced in patients with NCS compared with controls $(7323 \pm 689.1$ versus $8011 \pm 932.5 \mathrm{~mm}^{3}, P=.002$ ) (Fig 2). Volumes of the left rostral anterior cingulate cortex $(P=.014)$ and left insular cortex $(P=.018)$ were also reduced in patients compared with controls; however, the statistical significance of these 2 structures did not survive multiple comparisons by using a Bonferroni correction $(P<.00625)$.

Smaller right insular volumes in patients with NCS were related to larger drops in systolic $(r=-.410, P=.020)$ and diastolic $(r=-.507, P=.003)$ BP during HUT (Fig 3). There were no 

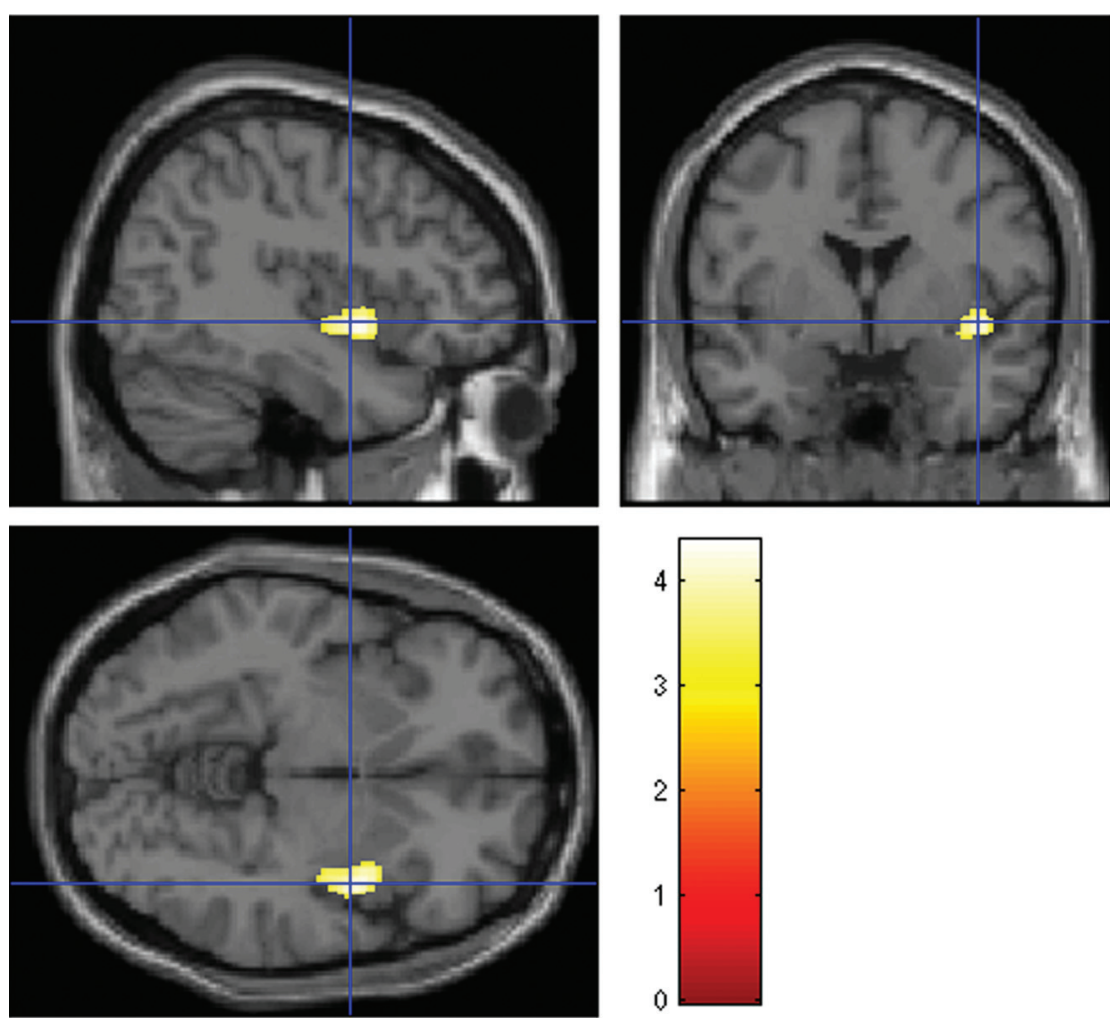

FIG 1. Statistical parametric maps showing regional differences in GM volume between 32 patients with NCS and 32 controls. Orthogonal images show a reduction of GM volume in the right insula in patients with NCS compared with controls, superimposed on a standard template $(P<.05$, cluster-level-corrected for multiple comparisons by using family-wise error). The color bar represents the $T$ values. The left side of each image is the left side of brain.

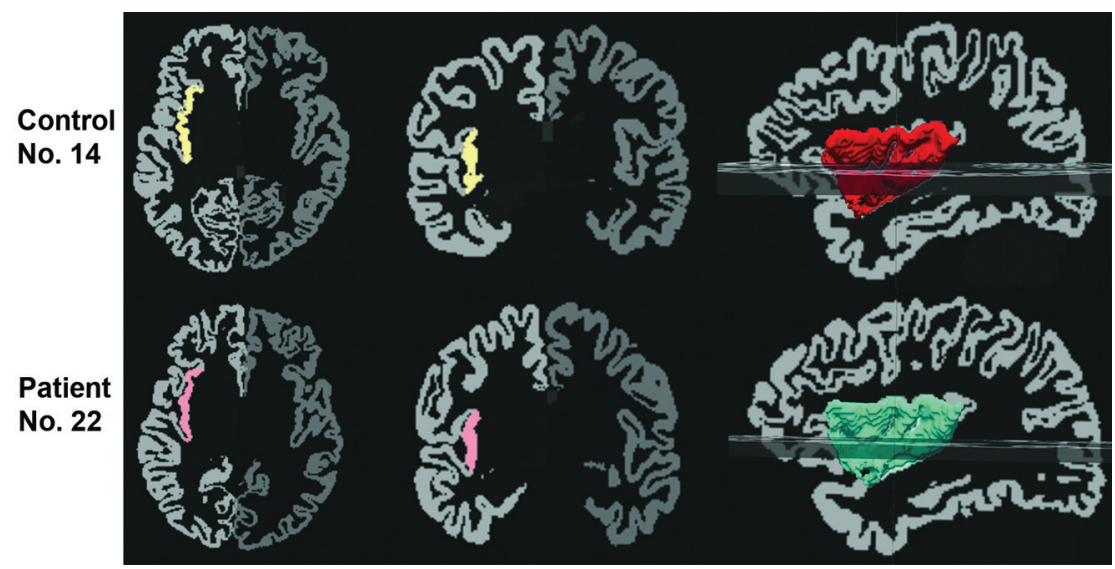

FIG 2. An illustration of segmentation and volume measurement of the right insula in a control subject (upper row, volume $=8119 \mathrm{~mm}^{3}$ ) and a patient (lower row, volume $=7247 \mathrm{~mm}^{3}$ ). The automatically segmented insular cortex is superimposed on axial (first column) and coronal (second column) GM images, and the third column illustrates a 3D reconstructed image of the right insula. The left side of each image is the right side of brain.

correlations between right insular volumes and other clinical variables including HR changes, disease duration, and number of total syncope and presyncope attacks (all $P>.05$ ). Volumes of the left rostral anterior cingulate and left insular cortices were not correlated with any clinical variables (all $P>.05)$.

\section{DISCUSSION}

This study attempted to explore cerebral structural changes associated with pathophysiologic mechanisms underlying NCS. Using
VBM, we found that patients with NCS had regional atrophy in the right insular cortex. We also observed a significant reduction of whole right insular volume in patients with NCS compared with controls, consistent with the VBM finding. Moreover, smaller right insular volumes in patients with NCS were related to larger drops in both systolic and diastolic BP during HUT, suggesting an important involvement of the right insular cortex in NCS.

Among the regions belonging to the central autonomic network, the insular cortex has been recognized as playing a crucial role in cardiovascular modulation..$^{20,21,24-26}$ An increase in nocturnal $\mathrm{BP}$ and occurrence of QT prolongation and cardiac arrhythmias were more frequently observed in patients with stroke with involvement of the insular cortex compared with those without insular involvement. ${ }^{27,28}$ Damage to the right insular cortex was associated with impairment in sympathovagal balance, supporting an important implication of the right insular cortex in autonomic control of cardiovascular activity. ${ }^{29,30}$ Several lines of evidence indicate that there is a hemispheric lateralization of cardiovascular autonomic control of the insular cortex. A study investigating heart rate variability changes during intracarotid amobarbital injection in patients with temporal lobe epilepsy showed sympathetic activation with left hemisphere injection and parasympathetic activation with right hemisphere injection. ${ }^{31}$ An intraoperative study before temporal lobectomy in patients with epilepsy demonstrated that BP and HR decreased with left insular stimulation, whereas BP and HR increased with right insular stimulation. ${ }^{24}$ These findings strongly support a critical role of the left insula in parasympathetic cardiovascular control and of the right insula in sympathetic control.

There is a reciprocal interaction between sympathetic and parasympathetic control of cardiac autonomic functions. ${ }^{32,33}$ Damage confined to the left insular cortex by acute stroke could shift cardiovascular balance toward an increase in basal sympathetic tone and a decrease in parasympathetic tone. ${ }^{34}$ Likewise, our finding could be interpreted as right insular atrophy in NCS being associated with a decrease in sympathetic activity and a reciprocal increase in parasympathetic activity, resulting in a drop in BP and syncope. Our speculation 

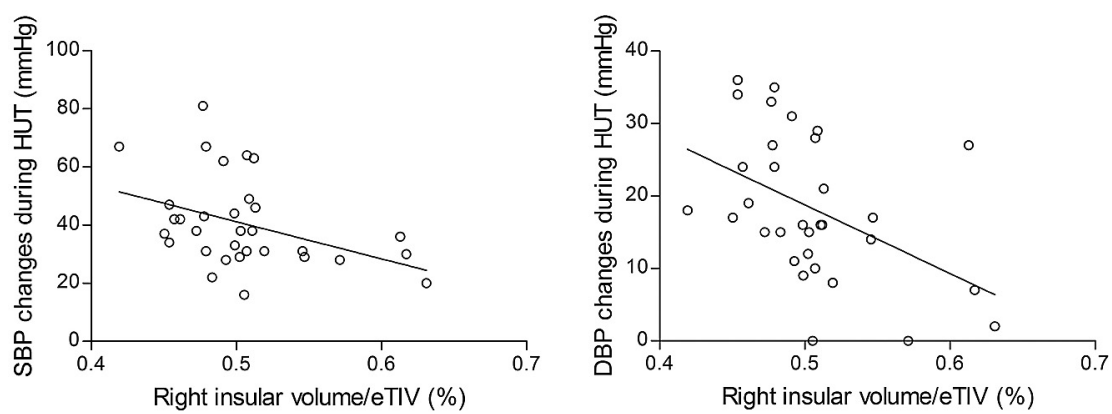

FIG 3. The relationship between the volume of the whole right insular cortex and BP changes in patients with NCS. Normalized right insular volume (raw volume/estimated total intracranial volume [eTIV] $\times 100$ ) was negatively correlated with the extent of drops in systolic BP (SBP) (left panel, $r=-.410, P=.020$ ) and diastolic BP (DBP) (right panel, $r=-.507, P=.003$ ) during the head-up tilt test (Spearman correlation, $P<.05$ ).

might be further supported by the findings of greater right insular volume reductions in relation to larger drops in both systolic and diastolic BP during HUT.

There are only a few studies evaluating hemodynamic and structural changes of the brain in patients with NCS. Studies by using SPECT showed decreases in regional cerebral blood flow in multiple cortical regions, yielding inconsistent results across the studies. ${ }^{35-37}$ The results of these SPECT studies might be interpreted as a consequence of cerebral hypoperfusion rather than a specific cause of NCS. To our knowledge, there is only 1 exploratory VBM study that showed a reduction of GM volume in the medulla in patients with NCS. The study suggested that NCS may be associated with structural changes of the medulla, which predisposes the patient to abnormal cardiovascular homeostasis and recurrent syncope. ${ }^{8}$ However, we did not observe any GM volume changes in the brain stem, even by using a lenient threshold of uncorrected $P<.01$. This difference could not be properly explained but might, in part, be ascribed to different sample sizes, MR scanners, and patient characteristics. Because a substantial number of conditions can mimic NCS, ${ }^{5}$ only patients with orthostatic stress and a positive response to HUT were included in our study. In their study, only 2 of 18 patients had orthostatic stress as a main trigger of syncope, and the remaining patients had variable triggers such as emotional distress, blood phobia, instrumentation, or pain. ${ }^{8}$ Because NCS comprises a heterogeneous group of conditions based on pathophysiologic classification of the principal triggers of syncope, ${ }^{4}$ the differences in the patient populations included might account for the inconsistency between the studies.

There are several limitations to our study. First, the current study is cross-sectional, and thus interpretation of our results with respect to a causal relationship is limited. Given that GM atrophy found in VBM could be a reflection of neuropathologic changes in many brain disorders such as temporal lobe epilepsy, Alzheimer disease, and schizophrenia, ${ }^{38}$ we hypothesized that right insular atrophy may represent a primary underlying pathology and therefore act as the specific cause that promotes NCS. Conversely, taken together with our finding of a trend toward significance for regional volume reductions of the left insula and anterior cingulate cortex in patients with NCS, it seems possible that right insular atrophy might be a consequence of repeated attacks of cerebral hypoperfusion. Further prospective study incorporating a longitudinal design would provide a hint of the causal relationship between insular atrophy and disease progression. Second, we did not measure heart rate variability, a representative indicator of autonomic cardiovascular functions that can quantify both sympathetic and parasympathetic activities. Therefore, examination of HUT without heart rate variability measurement may be insufficient to support our speculation that patients with NCS have decreased sympathetic activity and reciprocally increased parasympathetic activity.

\section{CONCLUSIONS}

We have observed a novel finding of right insular atrophy in patients with NCS with a positive response to HUT, implicating a role of right insular dysfunction in the pathophysiologic mechanism underlying NCS. Together with previous investigations, our findings suggest that right insular atrophy may be associated with a decrease in sympathetic activity and a reciprocal increase in parasympathetic activity, resulting in cerebral hypoperfusion and syncope. Confirmation of our findings in larger, more homogeneous datasets of patients with NCS is warranted.

Disclosures: Ji Hyun Kim—RELATED: Grant: This work was supported in part by the National Research Foundation of Korea Grant funded by the Korean Government (Grant No. 20100004827, 20110005418) and a Korea University Grant.

\section{REFERENCES}

1. Chen-Scarabelli C, Scarabelli TM. Neurocardiogenic syncope. $B M J$ 2004;329:336-41

2. Mathias CJ, Deguchi K, Schatz I. Observations on recurrent syncope and presyncope in 641 patients. Lancet 2001;357:348-53

3. Kaufmann H. Neurally mediated syncope: pathogenesis, diagnosis, and treatment. Neurology 1995;45(4 suppl 5):S12-18

4. Moya A, Sutton R, Ammirati F, et al. Guidelines for the diagnosis and management of syncope (version 2009). Eur Heart J 2009;30: 2631-71

5. McKeon A, Vaughan C, Delanty N. Seizure versus syncope. Lancet Neurol 2006;5:171-80

6. Abboud FM. Neurocardiogenic syncope. N Engl J Med 1993;328: $1117-20$

7. Mosqueda-Garcia R, Furlan R, Tank J, et al. The elusive pathophysiology of neurally mediated syncope. Circulation 2000;102: 2898-906

8. Beacher FD, Gray MA, Mathias CJ, et al. Vulnerability to simple faints is predicted by regional differences in brain anatomy. Neuroimage 2009; 47:937-45

9. Ashburner J, Friston KJ. Voxel-based morphometry: the methods. Neuroimage 2000;11:805-21

10. Kapoor WN, Smith MA, Miller NL. Upright tilt testing in evaluating syncope: a comprehensive literature review. Am J Med 1994;97: $78-88$

11. Grubb BP. Neurocardiogenic syncope and related disorders of orthostatic intolerance. Circulation 2005;111:2997-3006

12. Brignole M, Menozzi C, Gianfranchi L, et al. Carotid sinus massage, eyeball compression, and head-up tilt test in patients with syncope of uncertain origin and in healthy control subjects. Am Heart $J$ 1991;122:1644-51

13. Ashburner J, Friston KJ. Unified segmentation. Neuroimage 2005; 26:839-51

14. Dale AM, Fischl B, Sereno MI. Cortical surface-based analysis. I. 
Segmentation and surface reconstruction. Neuroimage 1999;9: 179-94

15. Fischl B, Salat DH, Busa E, et al. Whole brain segmentation: automated labeling of neuroanatomical structures in the human brain. Neuron 2002;33:341-55

16. Fischl B, van der Kouwe A, Destrieux C, et al. Automatically parcellating the human cerebral cortex. Cereb Cortex 2004;14:11-22

17. Ségonne F, Dale AM, Busa E, et al. A hybrid approach to the skull stripping problem in MRI. Neuroimage 2004;22:1060-75

18. Ségonne F, Pacheco J, Fischl B. Geometrically accurate topologycorrection of cortical surfaces using nonseparating loops. IEEE Trans Med Imaging 2007;26:518-29

19. Desikan RS, Segonne F, Fischl B, et al. An automated labeling system for subdividing the human cerebral cortex on MRI scans into gyral based regions of interest. Neuroimage 2006;31:968-80

20. Nagai $M$, Hoshide $S$, Kario $K$. The insular cortex and cardiovascular system: a new insight into the brain-heart axis. J Am Soc Hypertens 2010;4:174-82

21. Benarroch EE. The central autonomic network: functional organization, dysfunction, and perspective. Mayo Clin Proc 1993;68: 988-1001

22. Kimmerly DS, O'Leary DD, Menon RS, et al. Cortical regions associated with autonomic cardiovascular regulation during lower body negative pressure in humans. J Physiol 2005;569:331-45

23. Kurbaan AS, Franzén AC, Bowker TJ, et al. Usefulness of tilt testinduced patterns of heart rate and blood pressure using a two-stage protocol with glyceryl trinitrate provocation in patients with syncope of unknown origin. Am J Cardiol 1999;84:665-70

24. Oppenheimer SM, Gelb A, Girvin JP, et al. Cardiovascular effects of human insular cortex stimulation. Neurology 1992;42:1727-32

25. Oppenheimer S. Cerebrogenic cardiac arrhythmias: cortical lateralization and clinical significance. Clin Auton Res 2006;16:6-11

26. Williamson JW, Fadel PJ, Mitchell JH. New insights into central cardiovascular control during exercise in humans: a central command update. Exp Physiol 2006;91:51-58
27. Sander D, Klingelhofer J. Changes of circadian blood pressure patterns after hemodynamic and thromboembolic brain infarction. Stroke 1994;25:1730-37

28. Eckardt M, Gerlach L, Welter FL. Prolongation of the frequencycorrected QT dispersion following cerebral strokes with involvement of the insula of Reil. Eur Neurol 1999;42:190-93

29. Colivicchi F, Bassi A, Santini M, et al. Cardiac autonomic derangement and arrhythmias in right-sided stroke with insular involvement. Stroke 2004;35:2094-98

30. Tokgözoglu SL, Batur MK, Top uoglu MA, et al. Effects of stroke localization on cardiac autonomic balance and sudden death. Stroke 1999;30:1307-11

31. Yoon BW, Morillo CA, Cechetto DF, et al. Cerebral hemispheric lateralization in cardiac autonomic control. Arch Neurol 1997; 54:741-44

32. Kollai M, Koizumi K. Reciprocal and non-reciprocal action of the vagal and sympathetic nerves innervating the heart. J Auton Nerv Syst 1979;1:33-52

33. Malliani A, Pagani M, Lombardi F, et al. Cardiovascular neural regulation explored in the frequency domain. Circulation 1991;84: 482-92

34. Oppenheimer SM, Kedem G, Martin WM. Left-insular cortex lesions perturb cardiac autonomic tone in humans. Clin Auton Res 1996;6:131-40

35. Ilgin N, Olgunturk R, Kula $S$, et al. Brain perfusion assessed by 99mTc-ECD SPECT imaging in pediatric patients with neurally mediated reflex syncope. Pacing Clin Electrophysiol 2005;28:534-39

36. Töyry JP, Kuikka JT, Lansimies EA. Regional cerebral perfusion in cardiovascular reflex syncope. Eur J Nucl Med 1997;24:215-18

37. Joo EY, Hong SB, Lee M, et al. Cerebral blood flow abnormalities in patients with neurally mediated syncope. J Neurol 2011;258:366-72

38. Kakeda S, Korogi Y. The efficacy of a voxel-based morphometry on the analysis of imaging in schizophrenia, temporal lobe epilepsy, and Alzheimer's disease/mild cognitive impairment: a review. $\mathrm{Neu}$ roradiology 2010;52:711-21 